\title{
Eyelid swelling and erythema as the only signs of subperiosteal abscess
}

\author{
STEVEN E RUBIN, ${ }^{1}$ MICHAEL L SLAVIN, ${ }^{2}$ AND LORRY G RUBIN ${ }^{3}$
}

From the Divisions of 'Ophthalmology and ${ }^{3}$ Infectious Diseases of the Schneider Children's Hospital, and the Divisions of ${ }^{1}$ Pediatric Ophthalmology and Strabismus and ${ }^{2}$ Neuro-Ophthalmology of the Department of Ophthalmology of the Long Island Jewish Medical Center, New Hyde Park, New York and the Health Sciences Center, School of Medicine, State University of New York, Stony Brook, USA

SUMmARY On clinical grounds it is usually easy to distinguish between preseptal cellulitis, a cutaneous infection not threatening to vision, and orbital cellulitis, a potentially vision threatening infection of the orbital tissues generally arising from paranasal sinusitis. We recently cared for two patients with a clinical diagnosis of preseptal cellulitis who had CT scan evidence of subperiosteal abscess. Antibiotic therapy alone resulted in clinical resolution in each case.

Unilateral erythema and oedema of the eyelids unassociated with proptosis, conjunctival injection and chemosis, restricted motility, and diminished vision comprise the clinical diagnosis of preseptal cellulitis.' Radiological examination in such cases is infrequent, and empirical treatment with oral antibiotics on an ambulatory basis is often begun. More serious involvement of posterior structures (orbital

Correspondence to Steven E Rubin, MD, Ophthalmology Department, LIJMC, New Hyde Park, NY 11042, USA.

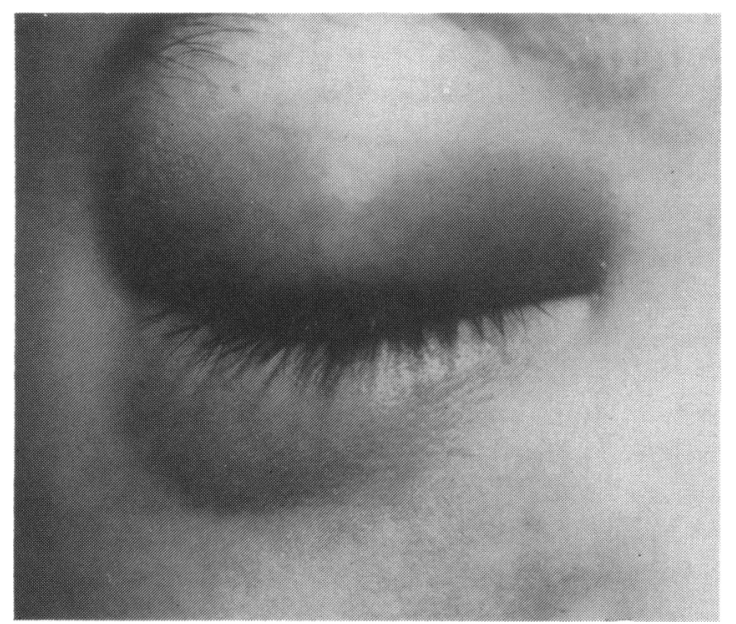

Fig. 1 Case 1. Note the marked oedema of upper and lower left eyelids. cellulitis), generally requiring intravenous therapy with admission to hospital, is usually indicated by proptosis, restricted motility, and/or visual dysfunction. ${ }^{1}$

We report on two patients with radiographic evidence of ethmoidal sinusitis and subperiosteal orbital abscess in whom eyelid swelling and erythema were the only clinical signs.

\section{Case reports}

CASE 1

A 4-year-old boy was well until he sustained unobserved (and presumed minor) trauma to his right eye. He developed transient conjunctival injection, which abated within 24 hours. One day later erythema and oedema of the right eyelids ensued. There was no accompanying fever or ocular pain.

Ophthalmic examination showed visual acuity of 6/9 (Allen pictures) in each eye. There was moderate oedema and slight erythema of the right upper and lower eyelids (Fig. 1). No chemosis or conjunctival injection was noted. The pupils were normal, with no afferent pupil defect. There was no proptosis or resistance to retropulsion of the globe. Extraocular movements were full, and he was orthophoric in primary gaze. Funduscopic examination showed nothing abnormal, and no choroidal folds were seen. The child was afebrile, and the general physical examination gave normal results. 


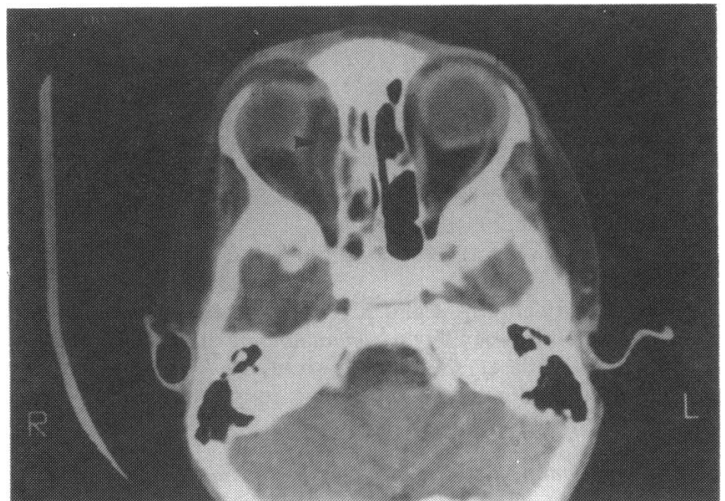

Fig. 2 Case 1. Computed axial tomography of the orbits showing subperiosteal elevation and lateral displacement of the medial rectus muscle (arrowhead).

Orbital computed tomography (CT) showed right ethmoidal sinusitis and a subperiosteal density with lateral displacement and enlargement of the medial rectus muscle (Fig. 2). No orbital fractures were identified. Intravenous antibiotic therapy (nafcillin and chloramphenicol) was instituted, and complete resolution of clinical signs was noted within one week. A follow-up CT scan performed two weeks later was normal.

\section{CASE 2}

A $71 / 2$-year-old boy had a four-day history of redness and swelling of his left eyelids accompanied by a left frontal headache. He experienced no change in his usual pattern of nasal discharge from chronic allergic rhinitis.

The boy was afebrile. His visual acuity was $6 / 6$ and

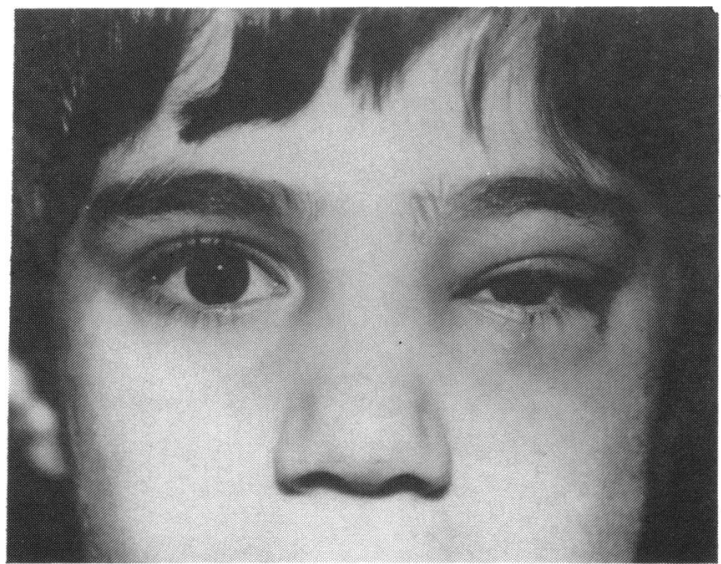

Fig. 3 Case 2. Preseptal oedema and erythema without conjunctival injection.

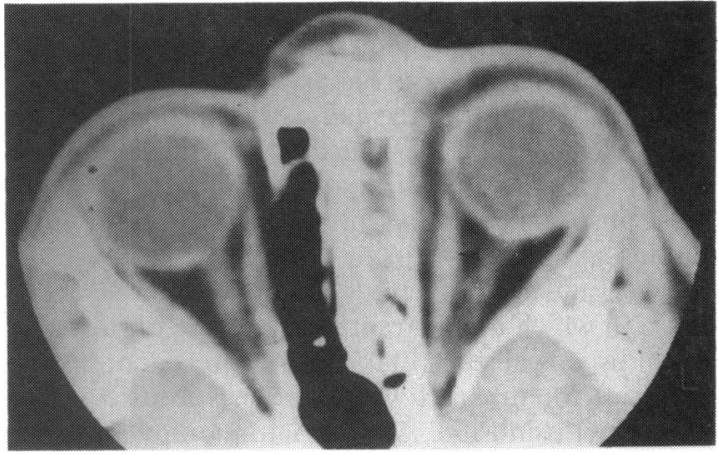

Fig. 4 Case 2. Computed axial tomography of the orbits showing ethmoid sinusitis and enlargement and lateral displacement of the left medial rectus muscle (arrowhead).

$6 / 7.5$ in the right and left eyes respectively. The left periorbital region was erythematous to the boundary of the orbital septum (Fig. 3). His pupils reacted normally, with no afferent pupillary defect. There was no strabismus, restricted motility, or proptosis, and no conjunctival injection or chemosis. Funduscopic examination gave normal results. CT scan revealed thickening of the periosteum on the left and bulging of the left medial rectus muscle into the muscle cone (Fig. 4).

The patient was treated with intravenous nafcillin and chloramphenicol for seven days and had complete clinical and radiological recovery.

\section{Discussion}

Isolated eyelid swelling and erythema usually indicates primary infection anterior to the orbital septum (preseptal cellulitis) ${ }^{1}$ but may also be the first sign of an underlying frontal or ethmoidal sinusitis ${ }^{2}$ (an association known better to otolaryngologists than ophthalmologists). While preseptal cellulitis (which usually results from minor trauma) may be treated with oral antibiotics on an ambulatory basis, orbital cellulitis and its subgroups, subperiosteal abscess and orbital abscess (which most often arise secondarily to an adjacent paranasal sinusitis), warrant more aggressive therapy with intravenous antibiotics and sometimes surgical intervention. ${ }^{34}$ The latter types of orbital infection are usually recognised because they produce proptosis and/or restricted motility. ${ }^{15}$ Unilateral, total, acute, irreversible visual loss was recently reported to occur in a patient on oral antibiotics with clinical paranasal sinusitis and isolated eyelid swelling as the only ophthalmic sign. ${ }^{6}$ None of the characteristic signs of orbital involvement were present (as in the two patients presented here). CT demonstrated spheno- 
ethmoiditis, with no evident orbital pathology. Presumably an optic neuropathy in the orbital apex or in the optic canal, secondary to adjacent infection or inflammation, was the cause of visual loss.

We consider that the clinical syndrome of preseptal cellulitis may overlap orbital cellulitis with subperiosteal abscess. Radiological exclusion of underlying paranasal sinusitis with or without orbital involvement should be considered, especially in cases such as these in which minor trauma as a cause of preseptal cellulitis was absent (making the antecedent history atypical), or in cases where there is a history consistent with sinusitis. Appropriate therapy may then be instituted to ward off possible visual sequelae.

\section{References}

1 Rubinstein JB, Handler SD. Orbital and periorbital cellulitis in children. Head Neck Surg 1982; 5: 15-21.

2 Chandler JR, Langenbrunner DJ, Stevens ER. The pathogenesis of orbital complications in acute sinusitis. Laryngoscope 1970; 80: 1414-27.

3 Hornblass A, Herschorn BJ, Stern D, Grimes C. Orbital abscess. Surv Ophthalmol 1984; 29: 169-78.

4 Bergin DJ, Wright JE. Orbital cellulitis. Br J Ophthalmol 1986; 70: $174-8$.

5 Weiss A, Friendly D, Eglin K, et al. Bacterial periorbital and orbital cellulitis in childhood. Ophthalmology 1983; 90: 195-203.

6 Slavin ML, Glaser JS. Acute severe irreversible visual loss with sphenoethmoiditis-'posterior' orbital cellulitis. Arch Ophthalmol 1987; 105: 345-8.

Accepted for publication 18 November 1988. 